\title{
Systematic review and meta-analysis of reduction in all-cause mortality from walking and cycling and shape of dose response relationship
}

Paul Kelly ${ }^{1,2^{*}}$, Sonja Kahlmeier ${ }^{3}$, Thomas Götschi ${ }^{3}$, Nicola Orsini ${ }^{4}$, Justin Richards ${ }^{5}$, Nia Roberts ${ }^{6}$, Peter Scarborough ${ }^{1}$ and Charlie Foster ${ }^{1}$

\begin{abstract}
Background and objective: Walking and cycling have shown beneficial effects on population risk of all-cause mortality (ACM). This paper aims to review the evidence and quantify these effects, adjusted for other physical activity (PA).
\end{abstract}

Data sources: We conducted a systematic review to identify relevant studies. Searches were conducted in November 2013 using the following health databases of publications: Embase (OvidSP); Medline (OvidSP); Web of Knowledge; CINAHL; SCOPUS; SPORTDiscus. We also searched reference lists of relevant texts and reviews.

Study eligibility criteria and participants: Eligible studies were prospective cohort design and reporting walking or cycling exposure and mortality as an outcome. Only cohorts of individuals healthy at baseline were considered eligible.

Study appraisal and synthesis methods: Extracted data included study population and location, sample size, population characteristics (age and sex), follow-up in years, walking or cycling exposure, mortality outcome, and adjustment for other co-variables. We used random-effects meta-analyses to investigate the beneficial effects of regular walking and cycling.

Results: Walking (18 results from 14 studies) and cycling (8 results from 7 studies) were shown to reduce the risk of all-cause mortality, adjusted for other PA. For a standardised dose of 11.25 MET.hours per week (or 675 MET. minutes per week), the reduction in risk for ACM was $11 \%(95 \% \mathrm{Cl}=4$ to $17 \%)$ for walking and $10 \%(95 \% \mathrm{Cl}=6$ to 13\%) for cycling. The estimates for walking are based on 280,000 participants and 2.6 million person-years and for cycling they are based on 187,000 individuals and 2.1 million person-years. The shape of the dose-response relationship was modelled through meta-analysis of pooled relative risks within three exposure intervals. The dose-response analysis showed that walking or cycling had the greatest effect on risk for ACM in the first (lowest) exposure interval.

Conclusions and implications: The analysis shows that walking and cycling have population-level health benefits even after adjustment for other PA. Public health approaches would have the biggest impact if they are able to increase walking and cycling levels in the groups that have the lowest levels of these activities.

(Continued on next page)

\footnotetext{
* Correspondence: paul.kelly@dph.ox.ac.uk

${ }^{1}$ Nuffield Department of Population Health, British Heart Foundation Centre on

Population Approaches for Non-Communicable Disease Prevention, WHO

Collaborating Centre on Population Approaches to Non-Communicable Disease

Prevention, University of Oxford, Oxford OX3 7LF, UK

${ }^{2}$ Physical Activity for Health Research Centre, Institute for Sport, Physical

Education and Health Sciences, University of Edinburgh, Edinburgh, UK

Full list of author information is available at the end of the article
} 
(Continued from previous page)

Review registration: The review protocol was registered with PROSPERO (International database of prospectively registered systematic reviews in health and social care) PROSPERO 2013: CRD42013004266.

Keywords: Walking, Cycling, Active travel, All-cause mortality

\section{Background}

Regular walking and cycling have become a key focus of physical activity (PA) promotion [1]. However, the expected public health benefit for different exposure levels on all-cause mortality (ACM) remains unclear. Most studies on PA and ACM have focussed on overall or leisuretime PA [2,3], and there have been relatively fewer studies reporting walking or cycling as the exposure [4]. Fewer still have reported the independent effect of walking or cycling, meaning results may be confounded by other PA. It may be that groups with high levels of walking or cycling do more leisure time or occupational PA which would inflate the health benefits attributed to these behaviours.

For public health policy, health impact assessment, comparative risk assessment, and health economic modelling, better evidence is required to address two questions: (1) what is the reduced risk for ACM from achieving PA recommendations through walking or cycling (adjusted for other PA); and (2) what is the shape of the dose-response curve across the range of exposures for walking and cycling?

When considering ACM and walking, previous metaanalyses have suggested a reduced risk of between 3 and $32 \%[2,3,5]$. This wide range is likely a function of non-standardised approaches to quantifying the exposure of walking and inclusion of studies that did not adjust for other PA. There has not previously been a meta-analysis of cycling and ACM, as sufficient evidence is only recently available [4].

Our first aim is to estimate the reduction of risk for ACM from walking and cycling using a robust method that would be of use to health modelling and risk assessment. We will standardise the exposures of walking and cycling in line with international PA recommendations and will only consider studies that adjusted for other PA. This will be of use to health modelling relying on basic walking and cycling data. In terms of the dose-response curve, it is increasingly accepted that the relationship between PA and health benefits may not be linear $[3,4,6]$. That is, a 30 minute per week increase in walking or cycling might result in benefits of differential magnitude if the existing baseline levels were, for example, 0 compared to 90 minutes per week. This is important for modelling change in behaviour as populations will not all have zero walking or cycling at baseline, and uptake is not always equitable [7]. Therefore, our second aim is to characterise the shape of the dose response relationships for walking and cycling across the range of exposures. This will be of use to health modelling efforts that have access to informative baseline, before and after, and differential uptake data.

\section{Research question 1}

What is the reduced risk of all-cause mortality from an exposure of (i) walking and (ii) cycling equivalent to physical activity recommendations (11.25 MET.hours per week)? (Population: identified cohort participants, healthy at baseline; Intervention: exposure to regular walking or cycling above referent level; Comparison: exposure to regular walking or cycling at referent (lowest) level; Outcome: all-cause mortality during follow up (controlled for other PA); Study design: prospective cohort studies).

\section{Research question 2}

What is the shape of the dose-response relationship for reduction in risk of all-cause mortality across the reported ranges of (i) walking and (ii) cycling exposures?

\section{Methods}

\section{Systematic review}

\section{Selection criteria}

The inclusion criteria for studies were as follows: prospective cohort design; healthy population at baseline (patient cohorts were excluded); exposure of regular walking or cycling at baseline was reported (and quantified by duration, distance or frequency); all-cause mortality rates, relative risks, or hazard ratios were reported (and adjusted for other physical activity). Only studies in English language were considered eligible.

\section{Search strategy}

We identified included studies in a 6 stage process. Searches were conducted in November 2013 using the following health databases of publications (Stage 1): Embase (OvidSP) (1974 - present); Medline (OvidSP) (1948-present); Web of Knowledge (1945 - present); CINAHL; SCOPUS; SPORTDiscus. The full search details (including search terms), identification, and data extraction strategy are available in the PROSPERO record (see Study protocol below). We also searched the reference lists of included studies and other topicrelated systematic reviews $[2-5,8]$ and contacted authors 
of all eligible studies for known grey literature and studies not published in peer-reviewed journals (Stage 2). One identified study originated from a book chapter and the lead author provided further details by email for data extraction that were not otherwise available. All records were assessed by two independent reviewers (PK and CF) at first and second appraisal (Stage 3 and 4) and any disagreements were resolved by discussion and mutual agreement. A third external researcher assessed $15 \%$ of excluded records at Stage 3 (contribution reported in acknowledgements). Detailed evaluation of full texts (Stage 5) was conducted independently (PK and $\mathrm{CF}$ ) with disagreements resolved by discussion with a third reviewer (SK). It was confirmed through author contact that studies from the same cohorts in conducted analysis on different subsets of individuals and therefore eligible for inclusion (Stage 6).

\section{Data extraction}

We collected data on study population, cohort name, study location, sample size, population characteristics (age and sex), follow-up (years and percentage), walking or cycling measures and reported exposures, outcome measures, other PA measures, reported outcomes (events, group size, relative risks or hazard ratios), and adjustment for other co-variables. Data extraction was performed by one researcher (PK). $25 \%$ of extracted data were cross-checked by an external researcher (contribution reported in acknowledgements).

\section{Quality assessment}

Study quality was assessed using a Newcastle Ottawa Scale (NOS) for Cohort Studies with appropriate selections made for primary controlling variable and length of follow up [9]. Two assessors (PK and JR) independently graded each study, and disagreements were resolved by discussion and mutual agreement. The NOS assessed: 1) representativeness of cohort, 2) selection of non-exposed cohort, 3) ascertainment of exposure, 4) demonstration that co-morbidities were not present at start of study, 5) control for main variable (age), 6) controls for any additional factors, 7) how outcome was ascertained, 8) follow-up time sufficient for outcome (5 years), and 9) percentage follow-up adequate. A study could score one point in each category with a possible total of nine points.

\section{Meta-analysis}

\section{Standardization of exposures}

The reported exposure levels of walking and cycling were converted into the same metric in order to standardise the magnitude of the exposures and calculate the associated risk reduction. We chose MET.hours per week for this metric as this allowed us to consider both the duration and the intensity of the reported exposures.
We adopted a conservative approach to convert the exposures into the common metric. For conversions, walking was defined to be 4.0 METs if not stated. This level was taken from the Compendium of Physical Activities (Taylor Code 015) and is described as "walking to work or class" [10]. Cycling was defined to be 6.8 METs if not stated. This level is described as "to/from work, selfselected pace" in the Compendium of Physical Activities [10]. These selections were based on the assumption that walking and cycling at a population level would be predominantly utilitarian i.e. active travel. The sensitivity of selecting different plausible values for walking and cycling was tested. If the exposure was reported as distance we assumed a walking speed of $3.3 \mathrm{mph}$ (or $5.3 \mathrm{~km} / \mathrm{h}$ ) to represent 4.0 METs [10] for conversions. If exposure was reported as sessions per week we assumed a session lasted 30 minutes, a method previously used by Woodcock et al. [3]. If exposures were reported as a range (e.g. 0 to 30 minutes per day) we took the midpoint of the range. If categories were open ended (e.g. more than 60 minutes per day) we assumed the range was the same width as the preceding range and estimated the midpoint accordingly.

\section{Calculating point estimates at 11.25 MET.hours per week}

Before constructing Forest plots, for each study we calculated point estimates of risk reduction at an exposure of walking or cycling equivalent to international PA recommendations. Current global recommendations for adults' PA are 150 minutes (2.5 hours) of moderate-intensity aerobic PA per week [11]; these recommendations define moderate intensity as 3 to 6 METs (metabolic equivalents) [11]. Taking the midpoint at 4.5 METs, we converted the recommendations to 11.25 MET.hours per week (the same metric as our converted exposures).

For each study we plotted reported risks against converted exposures and calculated the risk-estimate at 11.25 MET.hours per week. When referent categories were non-zero, we plotted additional MET.hours per week above baseline such that a relative risk of 1.0 equated to zero additional walking or cycling. For these calculations we assumed a linear relationship between walking and cycling and ACM risk. We also calculated point-estimates for other dose-response relationships by transforming the exposures of walking and cycling. Specifically, we tested the $\log$ and other various power transformations $(0.75$, $0.50,0.375,0.25)$ which have been theorised to represent the dose-response relationship between PA and ACM [3].

The confidence intervals for the point estimate at 11.25 MET.hours per week were calculated as the weighted mean of the standard errors for each of the exposure groups, extracted from the confidence intervals on reported relative risks. 


\section{Forest plots and funnel plots}

The pooled relative risks corresponding to $11.25 \mathrm{MET}$. hours per week were then estimated using randomeffects meta-analysis. This selection was made as included studies met the criteria for this approach [12].

Using the point estimates for risk reduction, random effects meta-analyses were performed using Stata version12 (StataCorp LP, College Station, Texas, USA) for walking and cycling. Variance in results that may be due to heterogeneity between studies was assessed using the $\mathrm{I}^{2}$ statistic. These data were displayed on Forest plots. Funnel plots were analysed to assess the possibility of small-study or publication bias.

\section{Dose-response relationship}

We chose to investigate the dose-response relationship through meta-analysis of pooled relative risks within three exposure intervals. A similar approach has been previously demonstrated for all PA and mortality [13]. Selection of the interval ranges (exposure categories) was based on the distribution of the reported walking and cycling exposures to give sufficient results in each category to allow meta-analysis. For walking, the categories for exposures in MET.hours per week were [1 to 16], [16 to 30] and [30 to 70]. For cycling the categories were [1 to 24], [24 to 40 ] and [40 to 90].

We then conducted separate random effects metaanalyses for each category using the risk reductions for exposures within the category range. When there was more than one exposure group from the same study within a single category we pooled the RR using the method proposed by Hamling et al. [14]. This method requires group size and number of events for each category. Included study authors were contacted by email to request missing data; studies were excluded from this analysis when these data were not available. When assessing the dose response relationship we were able to include results from 11/14 walking studies (79\%) and 6/7 cycling studies (86\%).

From these results we calculated the incremental change in risk for each MET.hour per week increase in either walking or cycling for the different regions of the exposure range. Finally the results of the separate meta-analyses were plotted against the midpoint of the respective exposure category. Confidence intervals were also plotted for each data point.

The analyses approaches are summarised in the analysis flow chart (Additional file 1: Figure S1).

\section{Study protocol}

The full study protocol was registered with PROSPERO (International database of prospectively registered systematic reviews in health and social care) and is available at http://www.crd.york.ac.uk/PROSPERO/display_record.asp? ID=CRD42013004266.

\section{Ethical approval}

Ethical approval was not required for this secondary analysis of cohort studies.

\section{Results}

\section{Systematic review}

A total of 8,901 titles were identified from databases, and 427 were retained after first appraisal with 4 further records identified from other sources. After second appraisal 49 full texts were retained for detailed evaluation with 18 meeting all inclusion criteria.

A number of identified walking studies were excluded from analysis after detailed evaluation: 18 did not adjust or control for other PA, 3 did not present ACM risk reductions (e.g. reported survival function), 3 did not report "walking only" on closer inspection (e.g. exposure was walking and standing), 3 reported walking by pace only preventing assessment of dose and 3 analysed the same participants as an already included higher quality study. We also excluded a number of studies that used walking as a dimension of PA (e.g. Low $=$ no PA, Medium $=$ Walking at least 3 hours per week, High $=$ Sports and exercise for 3 hours per week) as these did not allow assessment of the adjusted effect of walking. One small cycling study in older adults from the Netherlands matched the criteria for cycling and mortality, but was excluded as it did not control for other PA [15].

From the 18 included studies, 14 studies presented a total of 18 results on walking and ACM, and 7 studies presented a total of 8 results on cycling and ACM. These data are displayed in the study flow chart (Figure 1). Additional file 2: Table S1 shows the full data extraction for all included studies. Additional file 3: Table S2 shows the NOS quality scores.

\section{Description of studies Walking studies}

Of the 14 included studies, 7 came from Western Europe [16-21], 4 from the USA [22-25], 2 from China [26,27] and 1 from Japan [28]. The majority of results showed a reduced risk of ACM from walking, though only 5 from $18(28 \%)$ were statistically significant. The included studies contained 280000 individuals and 2.6 million person years. The aggregated mean age for participants was 56.6 years (range 20-93 years). The studies were generally high quality: 4 scored 9/9, 6 scored $8 / 9$ and 4 scored $7 / 9$ on the NOS.

\section{Cycling studies}

The majority of studies (6 of 7) came from Western Europe [16,29-33] (4 of which were from Denmark, 1 from UK and 1 from Germany), and 1 study came from China [27]. Cycling to work was the most common cycling domain assessed. Six studies showed either a statistically significant 


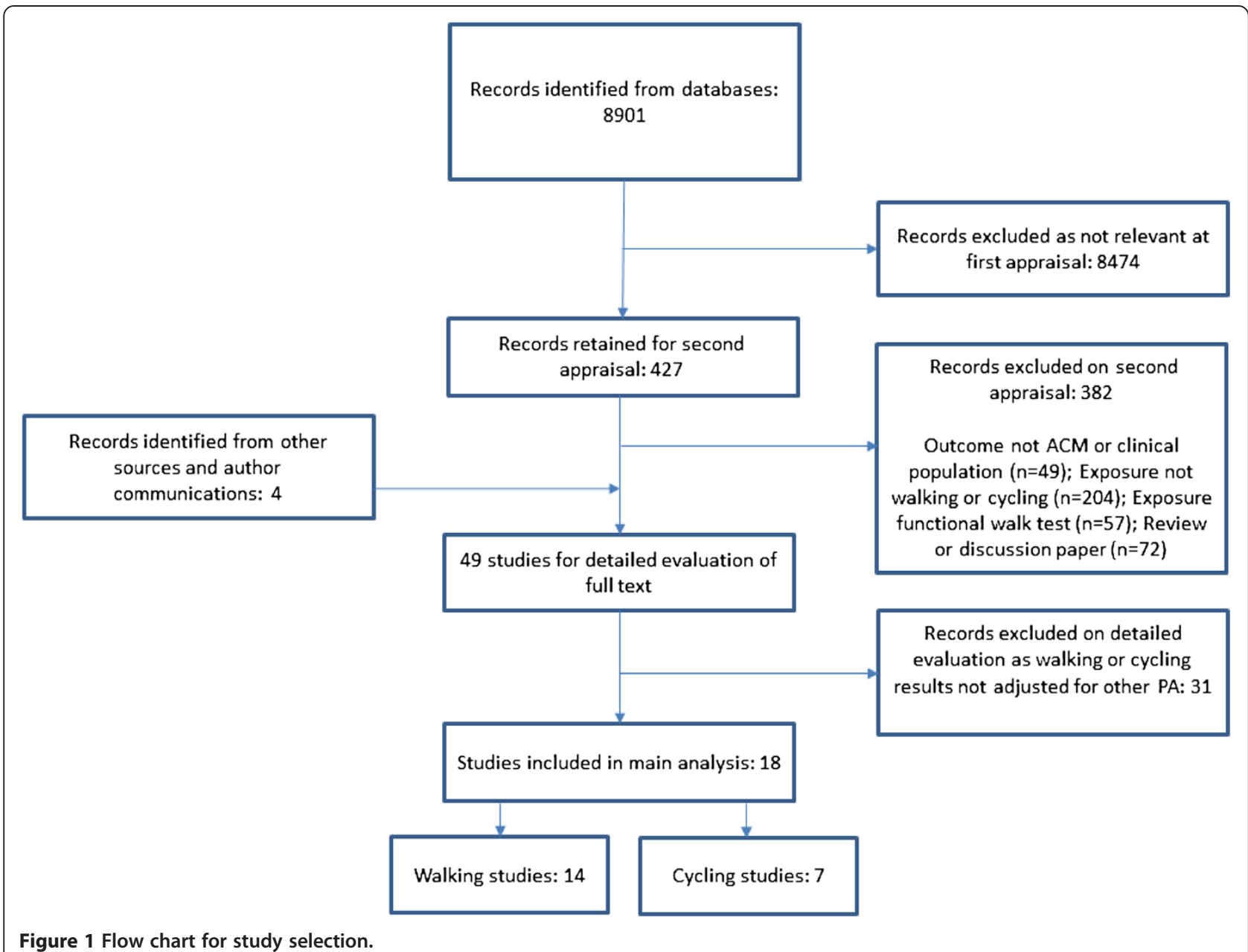

or a non-significant but still beneficial association between cycling and ACM. One study showed a small non-significant non-beneficial association between cycling and ACM [32].

The 7 studies for cycling contained 187000 individuals and 2.1 million person-years. The aggregated mean age for participants was 56.6 years (range 20-93 years) which was the same as the walking mean age and range. The studies were generally high quality: 2 scored 9/9, 2 scored 8/9, 2 scored 7/9 and 1 scored 6/9.

\section{Exposure conversions and point estimates}

Table 1 shows the included studies with the reported exposure categories and risk reductions. It also shows the conversion of reported exposure categories to MET. hours per week and derived point estimates for risk reduction at 11.25 MET.hours per week (with extracted 95\% CIs).

Once converted to point estimates at an exposure of 11.25 MET.hours per week, 3 of the 18 walking results (17\%) were statistically significant based on our extracted confidence intervals. For cycling, 4 of the 8 results $(50 \%)$ were significant at 11.25 MET.hours per week. There were no obvious patterns or differences between the results that were statistically significant and non-significant in terms of mean age, follow-up length, exposure ranges or study quality.

\section{Meta-analysis}

Random effects meta-analysis of the point estimates for the 18 results from 14 walking studies found a reduced risk of 0.89 ( $95 \% \mathrm{CI}=0.83$ to 0.96 ) corresponding to an exposure of 11.25 MET.hours per week (Figure 2). Random effects meta-analysis of point estimates for the 8 results from 7 cycling studies found a risk-reduction of 0.90 (95\% CI $=0.87-0.94)$ corresponding to an exposure to 11.25 MET.hours per week (Figure 3).

Sensitivity analyses on the selection of intensity for conversion of walking and cycling exposures were carried out and the calculations were found to be robust. For example, conducting the meta-analyses using an average intensity of 3.0 METs (instead of 4.0 
Table 1 Reported risks and calculated point estimates at 11.25 MET. hours per week by study

\begin{tabular}{|c|c|c|c|c|c|c|}
\hline Study - result & Reported exposure category & $\begin{array}{l}\text { Conversion to MET. } \\
\text { hours per week }\end{array}$ & $\begin{array}{l}\text { Additional MET. } \\
\text { hours per week }\end{array}$ & Reported risk & $\begin{array}{l}95 \% \text { confidence } \\
\text { interval }\end{array}$ & $\begin{array}{l}\text { Point estimate at } \\
\text { per week (extract }\end{array}$ \\
\hline \multicolumn{7}{|l|}{ Walking } \\
\hline \multirow[t]{2}{*}{ Johnsen (2013) [16] - Men } & Non-active & 0 & 0 & 1 & - & - \\
\hline & Active $=3$ hours $/$ week & 12 & 12 & 1.01 & 0.91 to 1.13 & $1.01(0.91$ to 1.12$)$ \\
\hline \multirow[t]{2}{*}{ Johnsen (2013) [16] - Women } & Non-active & 0 & 0 & 1 & - & - \\
\hline & Active $=3$ hours $/$ week & 12 & 12 & 0.92 & 0.80 to 1.06 & 0.93 (0.80 to 1.07$)$ \\
\hline \multirow[t]{2}{*}{ Wang (2013) [26] } & None & 0 & 0 & 1 & - & - \\
\hline & 5-6 hours/week@ 3.3 METs & 18.15 & 18.15 & 0.84 & 0.73 to 0.96 & 0.90 (0.79 to 1.03$)$ \\
\hline \multirow[t]{3}{*}{ Sabia (2012) [17] } & $<3.5$ hours/week & 0 & 0 & 1 & - & - \\
\hline & 3.5-5.9 hours/week & 12 & 12 & 0.83 & 0.63 to 1.08 & - \\
\hline & $6+$ hours/week & 22 & 22 & 0.81 & 0.61 to 1.07 & 0.89 (0.73 to 1.08$)$ \\
\hline \multirow[t]{2}{*}{ Nagai (2011) [28] - Men } & $<1$ hour/day & 14 & 0 & 1 & - & - \\
\hline & $>1$ hour/day & 42 & 28 & 0.9 & 0.82 to 0.98 & $0.96(0.88$ to 1.05$)$ \\
\hline \multirow[t]{2}{*}{ Nagai (2011) [28] - Women } & $<1$ hour/day & 14 & 0 & 1 & - & - \\
\hline & $>1$ hour/day & 42 & 28 & 0.95 & 0.82 to 1.10 & 0.98 (0.85 to 1.14$)$ \\
\hline \multirow[t]{3}{*}{ Stamatakis (2009) [18] - Men } & None & 0 & 0 & 1 & - & - \\
\hline & Med (2 sessions/week) & 4 & 4 & 1.1 & 0.86 to 1.14 & - \\
\hline & High (7 sessions/week) & 14 & 14 & 0.91 & 0.73 to 1.13 & 0.95 (0.84 to 1.08$)$ \\
\hline \multirow[t]{3}{*}{ Stamatakis (2009) [18] - Women } & None & 0 & 0 & 1 & - & - \\
\hline & Med (2 sessions/week) & 4 & 4 & 0.89 & 0.69 to 1.14 & - \\
\hline & High (7 sessions/week) & 14 & 14 & 0.92 & 0.72 to 1.18 & $0.92(0.77$ to 1.09$)$ \\
\hline \multirow[t]{3}{*}{ Besson (2008) [32] } & Non walker & 0 & 0 & 1 & - & - \\
\hline & Walk 0-90 min/week & 3 & 3 & 0.96 & 0.80 to 1.15 & - \\
\hline & Walk 90 min+/week & 9 & 9 & 0.89 & 0.73 to 1.09 & $0.86(0.78$ to 0.95$)$ \\
\hline \multirow[t]{4}{*}{ Matthews (2007) [27] } & 0-3.4 MET.hours/day & 11.9 & 0 & 1 & - & - \\
\hline & 3.5-7 MET.hours/day & 36.75 & 24.85 & 0.94 & 0.89 to 1.09 & - \\
\hline & 7.1-10 MET.hours/day & 59.5 & 47.6 & 0.83 & 0.69 to 1.00 & - \\
\hline & 10+ MET.hours/day & 81.2 & 69.3 & 0.86 & 0.71 to 1.05 & 0.97 (0.88 to 1.08$)$ \\
\hline \multirow[t]{4}{*}{ Schnohr (2007) [19] - Men } & $<0.5$ hours/day & 7 & 0 & 1 & - & - \\
\hline & $0.5-1.0$ hours/day & 21 & 14 & 0.87 & 0.68 to 1.10 & - \\
\hline & $1.0-2.0$ hours/day & 42 & 35 & 0.95 & 0.75 to 1.21 & - \\
\hline & $2.0+$ hours/day & 70 & 63 & 0.89 & 0.69 to 1.14 & 0.98 (0.85 to 1.13$)$ \\
\hline
\end{tabular}


Table 1 Reported risks and calculated point estimates at $\mathbf{1 1 . 2 5}$ MET. hours per week by study (Continued)

\begin{tabular}{|c|c|c|c|c|c|c|}
\hline \multirow[t]{4}{*}{ Schnohr (2007) [19] - Women } & $<0.5$ hours/day & 7 & 0 & 1 & - & - \\
\hline & $0.5-1.0$ hours/day & 21 & 14 & 1.00 & 0.77 to 1.30 & - \\
\hline & $1.0-2.0$ hours/day & 42 & 35 & 1.04 & 0.80 to 1.36 & - \\
\hline & $2.0+$ hours/day & 70 & 63 & 0.8 & 0.59 to 1.10 & 0.98 (0.83 to 1.15$)$ \\
\hline \multirow[t]{3}{*}{ Smith (2007) [22] } & None & 0 & 0 & 1 & - & - \\
\hline & $<1$ mile/day & 4.3 & 4.3 & 0.98 & 0.76 to 1.25 & - \\
\hline & $>1$ mile/day & 12.9 & 12.9 & 0.89 & 0.67 to 1.18 & 0.91 (0.75 to 1.10$)$ \\
\hline \multirow[t]{4}{*}{ Lee (2000) [23] } & $<5 \mathrm{~km} /$ week & 1.9 & 0 & 1 & - & - \\
\hline & 5 to $10 \mathrm{~km} /$ week & 5.8 & 3.9 & 0.91 & 0.82 to 1.02 & - \\
\hline & 10 to $20 \mathrm{~km} /$ week & 11.5 & 9.6 & 0.92 & 0.83 to 1.01 & - \\
\hline & $>20$ km/week & 19.2 & 17.3 & 0.84 & 0.75 to 0.94 & 0.89 (0.84 to 0.95$)$ \\
\hline \multirow[t]{2}{*}{ Bath (1998) [20] } & Less than 10 minutes/day & 2.3 & 0 & 1 & - & - \\
\hline & More than 10 minutes/day & 7 & 4.7 & 0.75 & 0.63 to 0.82 & 0.40 (0.34 to 0.48$)$ \\
\hline \multirow[t]{3}{*}{ Hakim (1998) [24] } & Less than 0.5 hour & 4.7 & 0 & 1 & - & - \\
\hline & $0.5-1.0$ hour & 14 & 9.3 & 0.66 & 0.47 to 0.91 & - \\
\hline & More than 1 hour & 46.7 & 42 & 0.55 & 0.37 to 0.83 & 0.87 (0.67 to 1.12 ) \\
\hline \multirow[t]{5}{*}{ Wannamethee (1998) [21] } & 0 min/day & 0 & 0 & 1 & - & - \\
\hline & 0-20 min/day & 4.7 & 4.7 & 1.15 & 0.73 to 1.79 & - \\
\hline & $21-40 \mathrm{~min} /$ day & 14 & 14 & 1.06 & 0.75 to 1.50 & - \\
\hline & 41-60 min/day & 23.3 & 23.3 & 0.97 & 0.65 to 1.46 & - \\
\hline & $60+\min /$ day & 32.7 & 32.7 & 0.62 & 0.37 to 1.05 & 0.93 (0.75 to 1.15 ) \\
\hline \multirow[t]{3}{*}{ La Croix (1996) [25] } & Less than 1 hour/week & 2 & 0 & 1 & - & - \\
\hline & 1-4 hours/wee & 10 & 8 & 0.83 & 0.53 to 1.29 & - \\
\hline & More than 4 hours/week & 22 & 20 & 0.91 & 0.58 to 1.42 & 0.92 (0.67 to 1.27 ) \\
\hline \multicolumn{7}{|l|}{ Cycling } \\
\hline \multirow[t]{2}{*}{ Johnsen (2013) [16] - Men } & Non-active & 0 & 0 & 1 & - & - \\
\hline & Active $=2$ hours $/$ week & 13.6 & 13.6 & 0.79 & 0.73 to 0.85 & 0.93 (0.87 to 0.99 ) \\
\hline \multirow[t]{2}{*}{ Johnsen (2013) [16] - Women } & Non-active & 0 & 0 & 1 & - & - \\
\hline & Active $=2$ hours $/$ week & 13.6 & 13.6 & 0.91 & 0.85 to 0.97 & 0.83 (0.77 to 0.89 ) \\
\hline \multirow[t]{3}{*}{ Sahlqvist (2013) [29] } & $0 \mathrm{~min} /$ week & 0 & 0 & 1 & - & - \\
\hline & 1-59 min/week & 3.4 & 3.4 & 0.96 & 0.78 to 1.17 & - \\
\hline & $60+\mathrm{min} /$ week & 10.2 & 10.2 & 0.91 & 0.84 to 0.99 & 0.90 (0.87 to 0.94 ) \\
\hline
\end{tabular}


Table 1 Reported risks and calculated point estimates at $\mathbf{1 1 . 2 5}$ MET. hours per week by study (Continued)

\begin{tabular}{|c|c|c|c|c|c|c|}
\hline & 0-3 hours/week & 10.2 & 10.2 & 0.78 & 0.69 to 0.88 & - \\
\hline & 3-7 hours/week & 24 & 34 & 0.76 & 0.68 to 0.85 & - \\
\hline & $7+$ hours/week & 61.2 & 61.2 & 0.7 & 0.62 to 0.78 & 0.94 (0.88 to 1.00$)$ \\
\hline \multirow[t]{9}{*}{ Schnohr (2010) [31] } & 15 min slow & 7 & 0 & 1 & - & - \\
\hline & 15 min ave & 11.9 & 4.9 & 0.67 & 0.49 to 0.92 & - \\
\hline & 15 min fast & 17.5 & 10.5 & 0.54 & 0.31 to 0.94 & - \\
\hline & 45 min slow & 21 & 14 & 0.87 & 0.57 to 1.33 & - \\
\hline & 45 min ave & 35.7 & 28.7 & 0.7 & 0.51 to 0.95 & - \\
\hline & 45 min fast & 52.5 & 45.5 & 0.44 & 0.28 to 0.69 & - \\
\hline & 75 min slow & 35 & 28 & 0.85 & 0.53 to 1.35 & - \\
\hline & 75 min ave & 59.5 & 52.5 & 0.71 & 0.52 to 0.57 & - \\
\hline & 75 min fast & 87.5 & 80.5 & 0.68 & 0.46 to 1.01 & 0.93 (0.80 to 1.07$)$ \\
\hline \multirow[t]{3}{*}{ Besson (2008) [32] } & Non cyclist & 0 & 0 & 1 & - & - \\
\hline & 0-30 min/week & 1.7 & 1.7 & 1.02 & 0.77 to 1.35 & - \\
\hline & $30+\mathrm{min} /$ week & 5.7 & 5.7 & 1.01 & 0.76 to 1.36 & 1.03 (0.84 to 1.26$)$ \\
\hline \multirow[t]{3}{*}{ Matthews (2007) [27] } & 0 MET.hours/day & 0 & 0 & 1 & - & - \\
\hline & 0.1-3.4 MET.hours/day & 12.3 & 12.3 & 0.79 & 0.61 to 1.01 & - \\
\hline & 3.5+ MET.hours/day & 36.1 & 36.1 & 0.66 & 0.40 to 1.07 & 0.88 (0.68 to 1.15$)$ \\
\hline \multirow[t]{2}{*}{ Andersen (2000) [33] } & No biking to work & 0 & 0 & 1 & - & - \\
\hline & Bike to work 3 hours/week & 20.4 & 20.4 & 0.72 & 0.57 to 0.91 & 0.85 (0.67 to 1.07$)$ \\
\hline
\end{tabular}




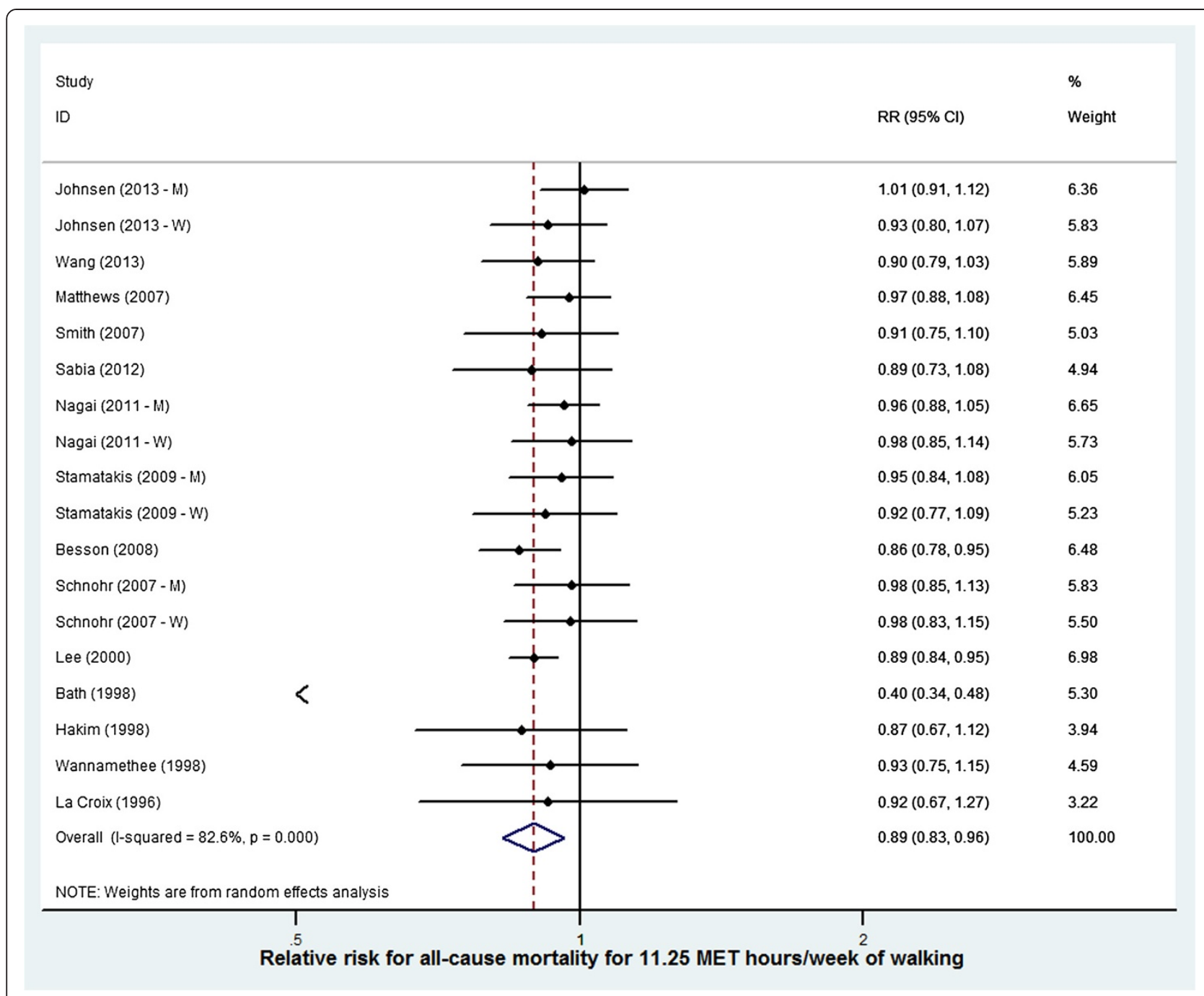

Figure 2 Risk reduction for all cause mortality for an additional 11.25 MET. hours per week of walking (studies displayed by quality score).

METs) for walking or 8.0 METs (instead of 6.8 METs) for cycling for the conversions made a 1 percentage point difference to the final meta-analyses results.

Likewise, when deriving point estimates of risk we tested various transformations to the exposure of walking and cycling to simulate non-linear dose-response relationships. Specifically we tested log-linear and various power transformations $(0.75,0.50,0.375,0.25)$. The walking meta-analysis varied by $1 \%(R R=0.89-0.90)$ and the cycling by $6 \%(R R=0.84-0.90)$ under these transformations. These data are shown in Table 2.

Heterogeneity in study results was assessed through examination of the forest plots and quantified using the $\mathrm{I}^{2}$ statistic to determine whether the observed variation in the study results was compatible with the variation expected by chance alone [12]. We used a randomeffects model as the default to incorporate heterogeneity between studies. $\mathrm{I}^{2}$ for walking was $82.6 \%(\mathrm{P}<0.05)$ and for cycling was $20.5 \%(\mathrm{p}=0.27)$ suggesting considerable heterogeneity.

We plotted trial effect against standard error and presented it as a funnel plot (Additional file 4: Figure S2). Asymmetry could be caused by a relationship between effect size and sample size or by publication bias, and we also examined any observed effect for heterogeneity [34]. We found no evidence of publication bias.

The study by Bath et al. [20] was a considerable outlier on the forest and funnel plots. By a substantial amount, this study had the greatest risk reduction at 11.25 MET. hours per week $(0.40$ ( $95 \% \mathrm{CI}=0.34$ to 0.48$)$; see Table 2). Repeating the analysis with Bath excluded did not change the pooled estimate for walking.

\section{Dose-response relationship}

The incremental change in risk for each MET.hour per week increase in either walking or cycling for the different 


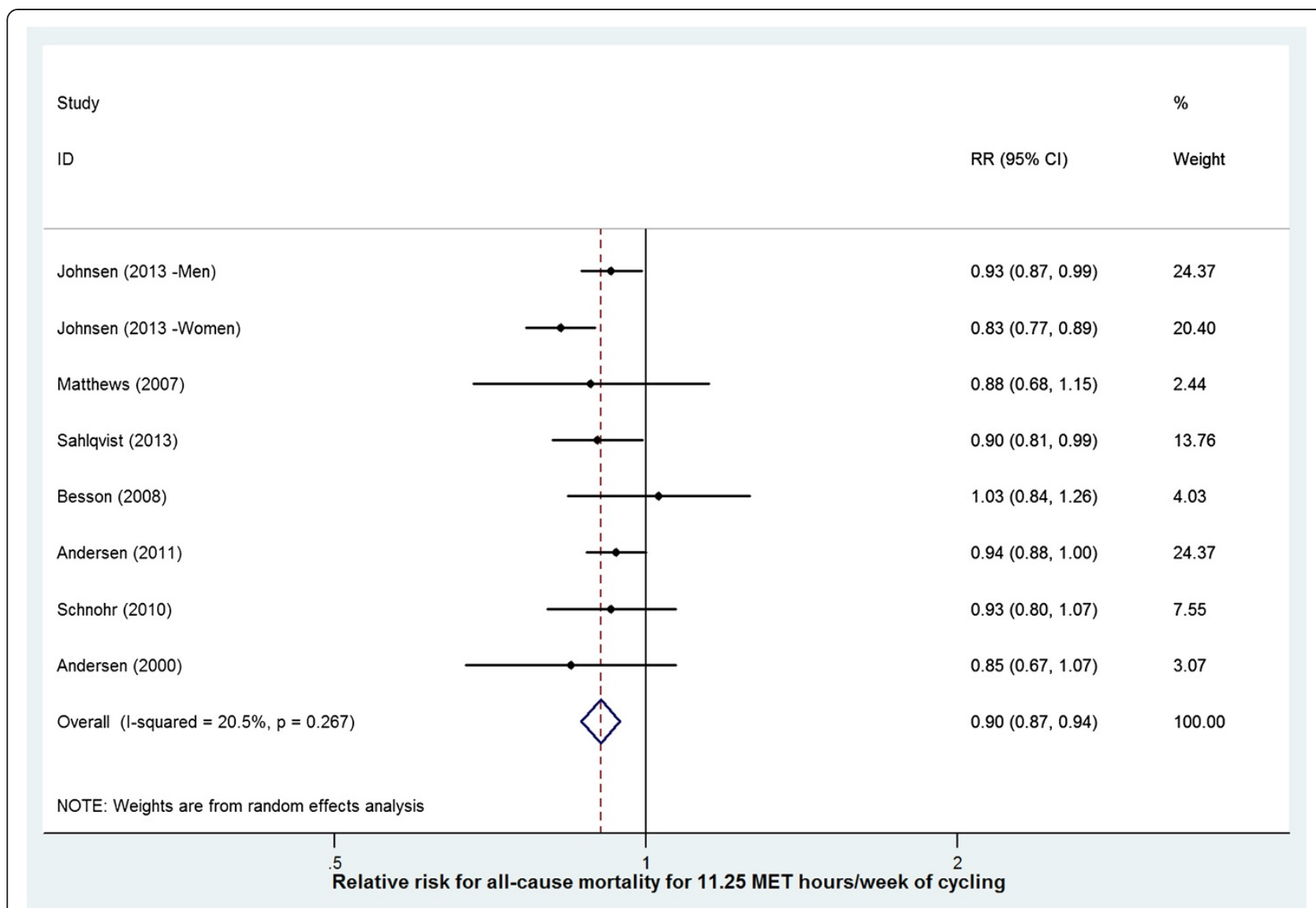

Figure 3 Risk reduction for all cause mortality for an additional 11.25 MET. hours per week of cycling (studies displayed by quality score).

exposure intervals are displayed in Table 3 . We found that the greatest rate of reduction is in the first category for both behaviours corresponding to 1-120 minutes per week of walking and 1-101 minutes per week of cycling.

Figures 4 and 5 show these same data as dose-response plots for walking and cycling across the ranges of reported exposures. Walking shows a slowing of the rate of benefit after 8 MET.hours per week (equivalent to 120 minutes per week at an intensity of 4.0 METs). The rate may increase slightly after 22.5 MET.hours per week, but the large confidence interval on the final data point suggests large uncertainty. The dose-response relationship for cycling shows the rate of received benefits drops in each subsequent category.

\section{Discussion}

The meta-analyses found an inverse association between both walking and cycling and risk of ACM. Including the most studies and person years, this is the largest walking meta-analysis and the first cycling meta-analysis to estimate the effect on ACM, adjusted for other physical activity. The results suggest that groups reporting an additional 11.25 MET.hours per week of walking (compared to groups reporting none or very little) reduce the risk of $\mathrm{ACM}$ by $11 \%$. For cycling the risk-reduction is $10 \%$. We tested the sensitivity of our analysis at 11.25 MET.hours per week to various dose-response relationships. The results were found to be robust at this exposure, particularly for walking.

We also investigated the shape of the dose-response relationship across the entire range of exposures (i.e. not limited to 11.25 MET.hours per week). Our analysis of these relationships suggested the largest effects in the first exposure category with decreasing rates of beneficial effects as the exposure to walking or cycling increased.

\section{Strengths and limitations}

The estimates for walking are based on 280000 participants and 2.6 million person-years and for cycling they are based on 187000 individuals and 2.1 million personyears. Further, the included studies were all high quality as assessed by the Newcastle-Ottawa scale. We only included studies that controlled for other domains of PA. This means the risk reductions calculated are more likely to be the result of walking or cycling exposure, independent of a confounding effect of other PA. However, the different ways and extents to which the studies 
Table 2 Meta-analysis results for different transformations simulating dose-response relationships

\begin{tabular}{llllll}
\hline & \multicolumn{2}{l}{ Walking } & & & Cycling \\
\cline { 2 - 3 } Dose-response & Relative risk & $\mathbf{9 5 \%} \mathbf{C l}$ & & Relative risk & $\mathbf{9 5 \% ~ C l}$ \\
\hline Linear & 0.89 & $0.83-0.96$ & & 0.90 & $0.87-0.94$ \\
Log-linear & 0.90 & $0.85-0.95$ & 0.90 & $0.86-0.94$ \\
0.75 power & 0.90 & $0.85-0.95$ & 0.89 & $0.85-0.93$ \\
0.50 power & 0.90 & $0.86-0.94$ & 0.87 & $0.83-0.91$ \\
0.375 power & 0.90 & $0.86-0.94$ & 0.86 & $0.81-0.91$ \\
0.25 power & 0.90 & $0.86-0.94$ & 0.84 & $0.79-0.90$ \\
\hline
\end{tabular}

Relative risks and $95 \%$ confidence interval estimated at $11.25 \mathrm{MET}$. hours per week.

assessed other PA prior to adjustment is a limitation to this method. The broad range of reported exposures also allowed us to investigate the dose-response relationships for walking and cycling across the distributions typically observed in general populations.

Included studies were predominantly from developed countries. It is not clear how generalizable the results would be to low and middle income countries where environmental or social factors might be different (e.g. road safety, air pollution, or prevalence of PA from other sources). While there was no evidence of publication bias in the funnel plots, we cannot completely rule out this possibility. Many studies were from large cohorts not specifically designed to test walking or cycling; it is possible other similar cohort studies finding non-significant or non-beneficial results did not publish their findings. The $\mathrm{I}^{2}$ statistic suggested considerable heterogeneity, especially for walking. This could be due to differences in exposure categorisation or sample bias, or due to residual confounding. The Bath study was a considerable outlier. It also had the one of the crudest categorisations of walking exposure (more or less than 10 minutes per day) which led to a large risk reduction when extrapolated to 11.25 MET.hours per week.

To convert the exposures to the same metric we had to make certain assumptions about intensity of behaviours and width of category ranges. These assumptions are needed as epidemiological studies on walking and cycling and ACM rarely capture distributions of speeds and intensities [19,31]. The intensity assumptions were reasonable and transparent; they are based on the widely used Compendium of Activities and the assumptions about mid-points of open ended ranges were necessary for our chosen approach. The results were shown to be robust to intensity selections. However, we must acknowledge that while required for our method, these assumptions and conversions may introduce some error. Further, at a population level walking and cycling will be described by a wide range of intensities based on individual fitness, journey purpose and terrain. For the meta-analysis of pooled relative risks, the choice of exposure categories is to some extent subjective and this is a known limitation of this approach.

The conversion of exposures to MET.hours per week and calculation of point estimates for risk is justified as this approach quantifies the exposure required for a given effect. This allows the reader to consider how much walking or cycling is required for a given effect and is a strength of our study.

As with many epidemiological studies, the measures of walking and cycling were often varied and crudely defined. In all cases they were self-reported. Our assumptions are unlikely to have added substantial further uncertainty; the exposure data allow us to estimate broad exposure to walking or cycling and the assumptions do not detract from this ability. Recent studies have also suggested that self-reported walking and cycling may be relatively valid at the group level [35]. We are confident that the findings are robust with regards to the methodological parameters available and selected for this analysis. The consistency of results across the studies, despite using different exposure levels and taking place in different walking and cycling environments support the overall conclusion of a beneficial effect of these forms of physical activity.

\section{Comparison to previous literature}

For walking a previous meta-analysis found a relative risk for $\mathrm{ACM}$ of 0.68 (95\% CI = 0.59 to 0.78 ) [5]. However this study did not specify or treat exposure in quantitative terms and thus it is not known how much walking confers this benefit. A meta-analysis of 13 studies by Samitz et al., reported a smaller reduced risk of 0.97 (95\% CI $=0.95$ to 0.99) for an increment one hour per week of walking [2].

Table 3 Incremental change in relative risk for 1 MET. hour per week increase in walking or cycling

\begin{tabular}{|c|c|c|c|c|c|c|}
\hline \multirow[b]{2}{*}{$\begin{array}{l}\text { Exposure } \\
\text { category }\end{array}$} & \multicolumn{3}{|l|}{ Walking } & \multicolumn{3}{|l|}{ Cycling } \\
\hline & $\begin{array}{l}\text { Exposure range } \\
\text { in MET. hours } \\
\text { per week }\end{array}$ & $\begin{array}{l}\text { Exposure range in } \\
\text { minutes per week } \\
\text { (@ } 4.0 \text { METs) }\end{array}$ & $\begin{array}{l}\text { Change in relative risk } \\
\text { for } 1 \text { MET. hour per } \\
\text { week increase }\left(x 10^{3}\right)\end{array}$ & $\begin{array}{l}\text { Exposure range } \\
\text { in MET. hours } \\
\text { per week }\end{array}$ & $\begin{array}{l}\text { Exposure range in } \\
\text { minutes per week } \\
\text { (@ } 6.8 \mathrm{METs} \text { ) }\end{array}$ & $\begin{array}{l}\text { Change in relative risk } \\
\text { for } 1 \mathrm{MET} \text {. hour per } \\
\text { week increase }\left(\mathrm{x} 10^{3}\right)\end{array}$ \\
\hline Category 1 & 0.1 to 8.0 & $1-120$ & 13.75 & 0.1 to 11.5 & $1-101$ & 14.78 \\
\hline Category 2 & 8.0 to 22.5 & $120-338$ & 2.07 & 11.5 to 32.0 & $101-282$ & 3.41 \\
\hline Category 3 & 22.5 to 50.0 & $338-750$ & 2.91 & 32.0 to 65.0 & $282-574$ & 1.82 \\
\hline
\end{tabular}




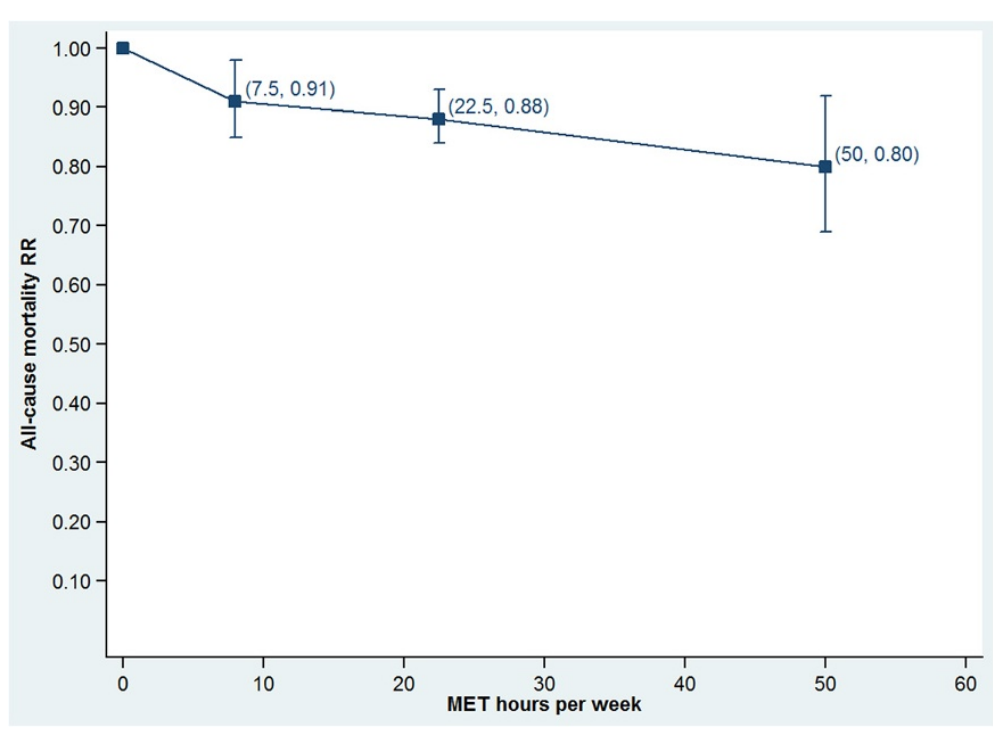

Figure 4 Dose-response relationship for walking across the range of reported exposures.

This is a lower exposure than we have used to calculate our risk reduction; extrapolated to our exposure this risk would be 0.92 which is $27 \%$ smaller than the magnitude of our finding. Neither analysis considered whether included studies had controlled for other PA.

A more recent meta-analysis of walking fitted a 0.375 power transformation to the exposure and standardised at 11 MET.hours per week. The authors reported a relative risk of 0.89 (95\% CI $=0.82$ to 0.96$)$ however they were only able to include five cohort studies (1.6 million person years) in this analysis [3]. They also included one study in which the exposure was a combination of walking and standing time [36]. Our results confirm this finding in a more robust analysis with new studies, more studies (all graded high quality) and covering 2.6 million person years.

A meta-analysis of cycling has not previously been possible due to the low number of published studies though Oja et al., and Saunders et al., have previously reviewed benefits for reduced morbidity from cycling $[4,8]$. This meta-analysis is helpful as individual studies have reported relative risks as low as 0.44 (95\% CI $=0.40$ to 1.07$)$ [31] and as high as $1.02(95 \% \mathrm{CI}=0.77$ to 1.35$)$ [32]. A meta-analysis of leisure-time physical activity (which might be considered similar to cycling) by Samitz et al., reported a risk reduction of $0.94(95 \% \mathrm{CI}=0.92$ -

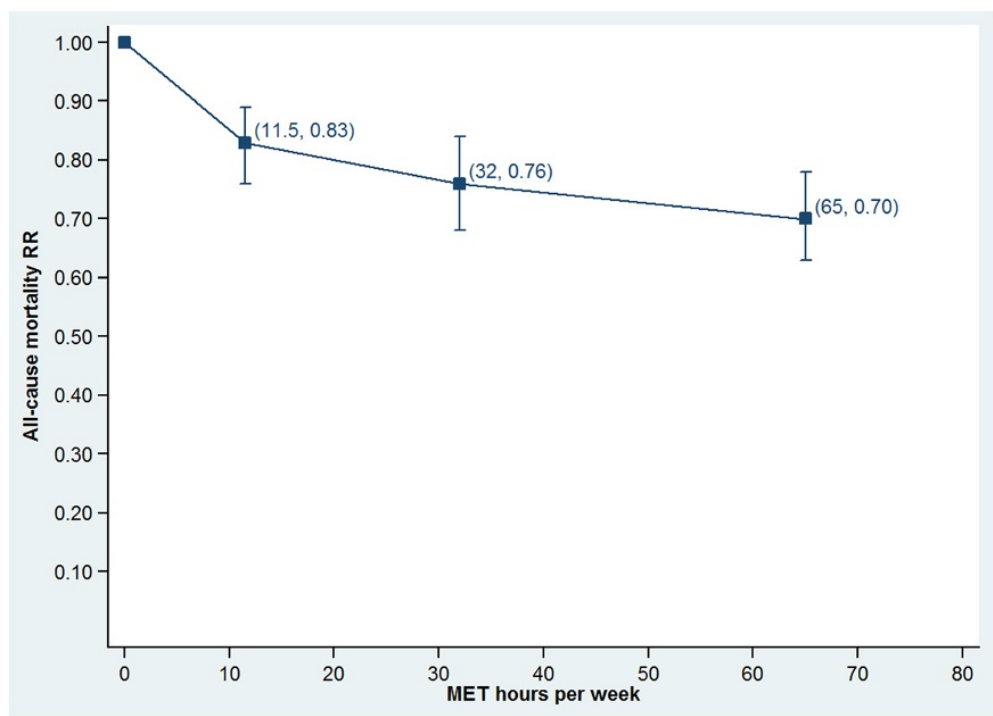

Figure 5 Dose-response relationship for cycling across the range of reported exposures. 
0.97) for an increment one hour of physical activity per week [2]. This analysis was based on seven studies, which did not all adjust for other PA. Extrapolated to our exposure this risk would be 0.90 which agrees with our finding.

It is now widely theorized that there is a non-linear dose-response relationship between various physical activities and reduced risk for all-cause mortality [3]. Woodcock presented a 0.375 power transform for walking and a 0.25 transform for non-vigorous PA. Oja et al. found evidence of an inverse step-wise decrease and a "ceiling effect" for general health benefit through increased cycling [4]. We chose to analyse the possible non-linear dose-response relationship through metaanalysis of pooled relative risks within three exposure intervals. This allowed us to better reflect the reported physiological effects at low and high exposures and the non-linear nature of the relationship between walking or cycling and ACM across the broad range of exposures. Another approach for modelling the quantitative exposure-response relationship is the use of cubic splines $[37,38]$ but many of our studies had dichotomous exposure variables, meaning they could not be included in such analysis and precluding this more advanced tool [37].

\section{Implications}

Previous walking analyses presented researchers with a wide range (3 to $32 \%$ ) of possible risk reductions. With our results, researchers can now model effects of walking and cycling, and compare to other risk factors, with greater confidence. Previous cycling modelling had to rely on results from single studies [39] and this new analysis will provide a better (and more conservative) estimate for researchers and modellers. We also present dose-response relationships for considering benefits across walking and cycling activity levels when before and after data are available to model change from different baseline exposures. Combined the results presented in this paper will provide a stronger information base for health impact assessment, economic analysis and active travel policy.

These results confirm that public health policy and recommendations should include walking and cycling. Walking in particular is an important behaviour as it is free, accessible and requires no equipment or training; it has been described as the nearest thing to "perfect exercise" [40]. Walking and cycling promotion strategies should also consider safety aspects to ensure that the positive health effects are not diminished by accidents in these more vulnerable road users [41]. The dose-response results suggest the greatest impact is in the first exposure category of walking and cycling per week. This is the first 120 minutes per week for walking and the first 100 minutes per week for cycling. Public health population approaches to PA promotion should focus on promotion and maintenance in this range for the greatest impact on ACM.

It may be considered unexpected that cycling, traditionally a more intense activity than walking had a very similar risk reduction (1 percentage point difference). We note that this is for the same energy expenditure, but as cycling is more intense, would be achieved in a shorter time (100 minutes per week for cycling; 170 minutes per week for walking).

Our methods have demonstrated a robust way to combine multiple studies with heterogeneous exposures in meta-analysis and estimate (1) the effect at PA recommendations and (2) the dose-response across the exposure range. Our paper also shows the need for improvement in exposure assessment. We have provided outputs that will be suitable for simple and complex modelling such as the Integrated Transport and Health Impact Modelling Tool (ITHIM) [42], the Health Economic Assessment Tool (HEAT) for walking and cycling $[39,43]$ or integrated models for preventable risk [44]. We have confirmed the beneficial effects and the greater importance of increasing the behaviours at low levels.

\section{Future research}

Systematic reviews generally report the need to update their results once new studies are published. We reserve judgement on this; the advent of cohort studies using objective exposure assessments such as accelerometer, pedometer or GPS (global positioning system) devices may indicate that subsequent reviews start afresh if exposures are non-comparable.

As more evidence does become available we recommend investigation of benefits and dose-response patterns by age, gender and geographic region. It is also important that studies of walking and cycling are conducted in low- and middle-income settings and included in any future iterations of this review to make the global utility of the findings more robust.

\section{Conclusions}

Walking and cycling confer $11 \%$ and $10 \%$ reductions in risk of ACM in groups that conduct 11.25 MET.hours per week. This effect is after adjustment for other PA. Analysis of dose-response suggests decreasing rate of benefit at higher exposures.

\section{Additional files}

Additional file 1: Figure S1. Summary of analysis approach.

Additional file 2: Table S1. Data extraction from included studies. Additional file 3: Table S2. Quality scores from modified newcastleottawa scale.

Additional file 4: Figure S2. Funnel plots for walking and cycling meta-analyses. 


\section{Abbreviations}

PA: Physical activity; ACM: All-cause mortality; METs: Metabolic equivalents; NOS: Newcastle Ottawa Scale.

\section{Competing interests}

The authors declare that they have no competing interests.

\section{Authors' contributions}

PK, SK, TG and CF conceived the study. JR and PK wrote the protocol. PK, JR and CF led title and full text screening and assessed study quality. PS and NO designed the analysis methods and supported PK in this stage. PK drafted the manuscript. All authors contributed to manuscript development and read and approved the final draft.

\section{Acknowledgements}

We acknowledge the support of the HEAT (Health Economic Assessment Tool) for Walking and Cycling International Advisory Groups for feedback on the study, the WHO Regional Office for Europe who provided financial support, Dr Aiden Doherty and Anja Mizdrak who supported study selection and data extraction and Dr Andrew Wright who provided statistical support.

\section{Author details}

${ }^{1}$ Nuffield Department of Population Health, British Heart Foundation Centre on Population Approaches for Non-Communicable Disease Prevention, WHO Collaborating Centre on Population Approaches to Non-Communicable Disease Prevention, University of Oxford, Oxford OX3 7LF, UK. ${ }^{2}$ Physical Activity for Health Research Centre, Institute for Sport, Physical Education and Health Sciences, University of Edinburgh, Edinburgh, UK. ${ }^{3}$ Institute of Social and Preventive Medicine, Physical Activity and Health, University of Zurich, $\mathrm{CH}-8001$ Zurich, Switzerland. ${ }^{4}$ Institute of Environmental Medicine, Karolinska Institutet, Stockholm, Sweden. ${ }^{5}$ Sydney Medical School \& Sydney School of Public Health, Charles Perkins Centre, The University of Sydney, Sydney NSW 2006, Australia. ${ }^{6}$ Bodleian Health Care Libraries, University of Oxford, Oxford, UK.

Received: 10 August 2014 Accepted: 13 October 2014 Published online: 24 October 2014

\section{References}

1. Bull FC, Gauvin L, Bauman A, Shilton T, Kohl HW 3rd, Salmon A: The Toronto charter for physical activity: a global call for action. J Phys Act Health 2010, 7:421-422.

2. Samitz G, Egger M, Zwahlen M: Domains of physical activity and all-cause mortality: systematic review and dose-response meta-analysis of cohort studies. Int J Epidemiol 2011, 40:1382-1400.

3. Woodcock J, Franco OH, Orsini N, Roberts I: Non-vigorous physical activity and all-cause mortality: systematic review and meta-analysis of cohort studies. Int J Epidemiol 2011, 40:121-138.

4. Oja P, Titze S, Bauman A, de Geus B, Krenn P, Reger-Nash B, Kohlberger T: Health benefits of cycling: a systematic review. Scand J Med Sci Sports 2011, 21:496-509.

5. Hamer M, Chida Y: Walking and primary prevention: a meta-analysis of prospective cohort studies. Br J Sports Med 2008, 42:238-243.

6. Sattelmair J, Pertman J, Ding EL, Kohl HW 3rd, Haskell W, Lee IM: Dose response between physical activity and risk of coronary heart disease: a meta-analysis. Circulation 2011, 124:789-795.

7. Ogilvie D, Foster CE, Rothnie H, Cavill N, Hamilton V, Fitzsimons CF, Mutrie N: Interventions to promote walking: systematic review. BMJ 2007, 334:1204.

8. Saunders LE, Green JM, Petticrew MP, Steinbach R, Roberts H: What are the health benefits of active travel? A systematic review of trials and cohort studies. PLoS One 2013, 8:e69912.

9. Wells GA, Shea B, O'Connell D, Peterson J, Welch V, Losos M, Tugwell P: The Newcastle-Ottawa Scale (NOS) for Assessing the Quality of Nonrandomised Studies in Meta-analyses. Ottawa, ON: Ottawa Hospital Research Institute; 2012.

10. Ainsworth BE, Haskell WL, Herrmann SD, Meckes N, Bassett DR Jr, TudorLocke C, Greer JL, Vezina J, Whitt-Glover MC, Leon AS: Compendium of physical activities: a second update of codes and MET values. Med SCi Sports Exerc 2011, 2011(43):1575-1581.

11. World Health Organisation: Global Recommendations on Physical Activity for Health. Geneva: World Health Organisation; 2010.

12. Deeks JJ, Higgins JPT, Altman DG: Analysing Data and Undertaking Meta-Analyses. In Cochrane Handbook for Systematic Reviews of
Interventions. John Wiley \& Sons, Ltd; 2008:243-296. http://handbook.cochrane. org/chapter_9/9_analysing_data_and_undertaking_meta_analyses.htm.

13. Committee PAGA: Physical activity guidelines advisory committee report, 2008. Washington, DC: US Department of Health and Human Services 2008, 2008:143-145.

14. Hamling J, Lee $P$, Weitkunat R, Ambuhl M: Facilitating meta-analyses by deriving relative effect and precision estimates for alternative comparisons from a set of estimates presented by exposure level or disease category. Stat Med 2008, 27:954-970.

15. Bijnen FC, Feskens EJ, Caspersen CJ, Nagelkerke N, Mosterd WL, Kromhout D: Baseline and previous physical activity in relation to mortality in elderly men: the Zutphen Elderly Study. Am J Epidemio/ 1999, 150:1289-1296.

16. Johnsen NF, Ekblond A, Thomsen BL, Overvad K, Tjonneland A: Leisure time physical activity and mortality. Epidemiology 2013, 24:717-725.

17. Sabia S, Dugravot A, Kivimaki M, Brunner E, Shipley MJ, Singh-Manoux A: Effect of intensity and type of physical activity on mortality: results from the Whitehall II cohort study. Am J Public Health 2012, 102:698-704.

18. Stamatakis E, Hamer M, Lawlor DA: Physical activity, mortality, and cardiovascular disease: is domestic physical activity beneficial? The Scottish Health Survey-1998, and 2003. Am J Epidemiol 2009, 169:1191-1200.

19. Schnohr P, Scharling H, Jensen JS: Intensity versus duration of walking, impact on mortality: the Copenhagen City Heart Study. Eur J CardiovasC Prev Rehabil 2007, 14:72-78.

20. Bath PA, Morgan K: Customary physical activity and physical health outcomes in later life. Age Ageing 1998, 27(Suppl 3):29-34.

21. Wannamethee SG, Shaper AG, Walker M: Changes in physical activity, mortality, and incidence of coronary heart disease in older men. Lancet 1998, 351:1603-1608.

22. Smith TC, Wingard DL, Smith B, Kritz-Silverstein D, Barrett-Connor E: Walking decreased risk of cardiovascular disease mortality in older adults with diabetes. J Clin Epidemiol 2007, 60:309-317.

23. Lee IM, Paffenbarger RS Jr: Associations of light, moderate, and vigorous intensity physical activity with longevity. The Harvard Alumni Health Study. Am J Epidemiol 2000, 151:293-299.

24. Hakim AA, Petrovitch $H$, Burchfiel CM, Ross GW, Rodriguez BL, White LR, Yano K, Curb JD, Abbott RD: Effects of walking on mortality among nonsmoking retired men. N Engl J Med 1998, 338:94-99.

25. LaCroix AZ, Leveille SG, Hecht JA, Grothaus LC, Wagner EH: Does walking decrease the risk of cardiovascular disease hospitalizations and death in older adults? J Am Geriatr Soc 1996, 44:113-120.

26. Wang N, Zhang X, Xiang YB, Li H, Yang G, Gao J, Zheng W, Shu XO: Associations of Tai Chi, walking, and jogging with mortality in Chinese men. Am J Epidemiol 2013, 178:791-796.

27. Matthews CE, Jurj AL, Shu XO, Li HL, Yang G, Li Q, Gao YT, Zheng W: Influence of exercise, walking, cycling, and overall nonexercise physical activity on mortality in Chinese women. Am J Epidemiol 2007, 165:1343-1350.

28. Nagai M, Kuriyama S, Kakizaki M, Ohmori-Matsuda K, Sone T, Hozawa A, Kawado M, Hashimoto S, Tsuji I: Impact of walking on life expectancy and lifetime medical expenditure: the Ohsaki Cohort Study. BMJ Open 2011, 1:e000240.

29. Sahlqvist S, Goodman A, Simmons RK, Khaw KT, Cavill N, Foster C, Luben R, Wareham NJ, Ogilvie D: The association of cycling with all-cause, cardiovascular and cancer mortality: findings from the population-based EPIC-Norfolk cohort. BMJ Open 2013, 3:e003797.

30. Andersen LB, Cooper AR: Commuter Cycling and Health. In Transport and Health Issues 2011: Studies on Mobility and Transport Research. Volume 3. Edited by Gronau W, Reiter K, Pressl R. Mannheim, Tyskland: Verlag MetaGISInfosysteme; 2011:9-19.

31. Schnohr P, Marott JL, Jensen JS, Jensen GB: Intensity versus duration of cycling, impact on all-cause and coronary heart disease mortality: the Copenhagen City Heart Study. Eur J Prev Cardiol 2012, 19:73-80.

32. Besson H, Ekelund U, Brage S, Luben R, Bingham S, Khaw KT, Wareham NJ: Relationship between subdomains of total physical activity and mortality. Med Sci Sports Exerc 2008, 40:1909-1915.

33. Andersen LB, Schnohr P, Schroll M, Hein HO: All-cause mortality associated with physical activity during leisure time, work, sports, and cycling to work. Arch Intern Med 2000, 160:1621-1628.

34. Sterne JAC, Egger M, Moher D: Addressing Reporting Biases. In Cochrane Handbook for Systematic Reviews of Interventions. John Wiley \& Sons, Ltd; 2008:297-333. 
35. Kelly P, Doherty A, Mizdrak A, Marshall S, Kerr J, Legge A, Godbole S, Badland $\mathrm{H}$, Oliver M, Foster C: High group level validity but high random error of a self-report travel diary, as assessed by wearable cameras. $J$ Transport Health 2014, 1:190-201.

36. Inoue M, Iso H, Yamamoto S, Kurahashi N, Iwasaki M, Sasazuki S, Tsugane S: Daily total physical activity level and premature death in men and women: results from a large-scale population-based cohort study in Japan (JPHC study). Ann Epidemiol 2008, 18:522-530.

37. Orsini N, Bellocco R, Greenland S: Generalized least squares for trend estimation of summarized dose-response data. Stata J 2006, 6:40-57.

38. Chau JY, Grunseit AC, Chey T, Stamatakis E, Brown WJ, Matthews CE, Bauman AE, van der Ploeg HP: Daily sitting time and All-cause mortality: a meta-analysis. PLoS One 2013, 8:e80000.

39. Rutter H, Cavill N, Racioppi F, Dinsdale H, Oja P, Kahlmeier S: Economic impact of reduced mortality due to increased cycling. Am J Prev Med 2013, 44:89-92.

40. Morris JN, Hardman AE: Walking to health. Sports Med 1997, 23:306-332

41. de Nazelle A, Nieuwenhuijsen MJ, Anto JM, Brauer M, Briggs D, BraunFahrlander C, Cavill N, Cooper AR, Desqueyroux H, Fruin S, Hoek G, Panis Ll, Janssen N, Jerrett M, Joffe M, Andersen ZJ, van Kempen E, Kingham S, Kubesch N, Leyden KM, Marshall JD, Matamala J, Mellios G, Mendez M, Nassif H, Ogilvie D, Peiró R, Pérez K, Rabl A, Ragettli M, et al: Improving health through policies that promote active travel: a review of evidence to support integrated health impact assessment. Environ Int 2011, $37: 766-777$

42. Woodcock J, Givoni M, Morgan AS: Health impact modelling of active travel visions for england and wales using anIntegrated Transport and Health Impact Modelling Tool (ITHIM). PLoS One 2013, 8:e51462.

43. Kahlmeier S, Racioppi F, Cavill N, Rutter H, Oja P: "Health in all policies" in practice: guidance and tools to quantifying the health effects of cycling and walking. J Phys Act Health 2010, 7(Suppl 1):S120-S125.

44. Scarborough P, Nnoaham KE, Clarke D, Capewell S, Rayner M: Modelling the impact of a healthy diet on cardiovascular disease and cancer mortality. J Epidemiol Commun Health 2012, 66:420-426

doi:10.1186/s12966-014-0132-x

Cite this article as: Kelly et al: Systematic review and meta-analysis of reduction in all-cause mortality from walking and cycling and shape of dose response relationship. International Journal of Behavioral Nutrition and Physical Activity 2014 11:132.

\section{Submit your next manuscript to BioMed Central and take full advantage of:}

- Convenient online submission

- Thorough peer review

- No space constraints or color figure charges

- Immediate publication on acceptance

- Inclusion in PubMed, CAS, Scopus and Google Scholar

- Research which is freely available for redistribution 